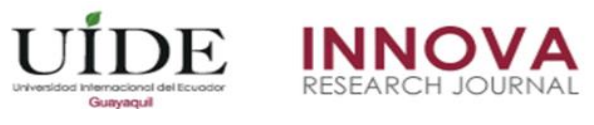

INNOVA Research Journal, ISSN 2477-9024

(Febrero, 2017). Vol. 2, No.2 pp.1-13

DOI: https://doi.org/10.33890/innova.v2.n2.2017.112

URL: http://revistas.uide.edu.ec/index.php/innova/index

Correo: innova@uide.edu.ec

\title{
Oportunidad de emprendimiento con responsabilidad ambiental a partir de fibras sintéticas y pieles artificiales en el Ecuador
}

\section{Opportunity for entrepreneurship with environmental responsibility from synthetic fibers and artificial skins in Ecuador}

Nelly Sayenka Vergara Díaz

Universidad de Guayaquil, Ecuador

Fernando Rodolfo Orellana Intriago

Universidad de Guayaquil, Ecuador

Víctor Hugo Vizueta Tomalá

Francisco Xavier Mata López

David Alberto Bernal Paredes

Zila Isabel Esteves Fajardo

Diana Carolina Hidalgo Seminario

Autor para correspondencia: nellysvergarad@gmail.com, fernando.orellanai@ug.edu.ec, vizuetavh@hotmail.com,presimaster1@hotmail.com, davidbernalparedes@gmail.com, zila.estevesf@ug.edu.ec, dicarolina.hidalgo88@ hotmail.com.

Fecha de recepción: 06 de Noviembre de 2016 - Fecha de aceptación: 10 de Enero de 2017

Resumen: El (Censos, 2016) (INEC), en su encuesta nacional de empleo, desempleo y subempleo en el Ecuador, de marzo de 2016, informó que el 68,6\% de las personas en edad de trabajar se considera económicamente activo; de éstos, el 94,3\% se consideran con empleo, el 5,7\% no cuenta con empleo formal o independiente. De las personas en edad de trabajar 31,4\% forman parte de la población económicamente inactiva. El emprendimiento se presenta como una solución que permite generar empleo. A la luz de nuevas investigaciones sobre el consumo de carnes y productos procesados. Está evolucionando las percepciones de la población y se están modificando sus hábitos. Se cuestionan más sobre los efectos en su salud y sobre el impacto ambiental, es mayor la demanda de productos en cuya elaboración se genere un menor nivel de impacto ambiental. Siendo la responsabilidad ambiental, un factor que a las empresas competitivas globales y locales las ha llevado no sólo a cumplir con las disposiciones legales, sino a crear ventajas competitivas a partir de la promoción del trabajo realizado en favor de la protección del medio ambiente. El fomento de emprendimientos con responsabilidad ambiental, mediante el uso de fibras sintéticas y pieles artificiales ayudará a generar herramientas para incrementar el empleo, generando valor con responsabilidad ambiental.

Palabras clave: emprendimiento; responsabilidad ambiental; exportación; fibras artificiales

Abstract: The (Census, 2016) (INEC), in its national survey of employment, unemployment and underemployment in Ecuador on march-2016, informed that $68,6 \%$ of people that have the age to work are considered economically active; of these ones, the $94,3 \%$ are employed, 5,7\% don't have formal employment o independent. From people that have the age to work, 31, $4 \%$ belong to economically inactive population. Entrepreneurship is the solution that develops employment. Through new light investigations about the meat consumption and processed products. The perception of population is evolving and their habits are being modified. The effects on health and environmental impacts are questioned. It's higher the demand of products which elaboration generates a lower environment impact. Being the environmental responsibility, a factor that competitive global and local companies has motivated them not only to obey legal dispositions, also to create competitive advantages through the job promotion realized to protect the natural environment. The phenomenon of ventures with environmental responsibility, through the use of synthetic fibers and artificial skins will help to generate tools to increase employment and develop value with social responsibility.

Key Words: Venture; enviromental responsibility; exports; artificial fibers 


\section{Introducción}

En Ecuador los años de crecimiento económico de la década del 2000, en conjunto con el boom del elevado precio de petróleo condición circunstancial de los mercados internacionales, paralelamente se dio pasos a dinamizar la economía basados en la construcción y actividades conexas ha supuesto el abandono de actividades tradicionales del ámbito rural y el trasvase de mano de obra del ámbito rural al urbano, provocando que el relevo generacional en estas actividades resultara casi inexistente. Este modelo de crecimiento centrado en el urbanismo, ahora fracasado, ha dejado un elevado número de trabajadores sin trabajo y, lo que es peor, sin expectativas claras de hacía donde orientar sus esfuerzos. Esta coyuntura, requiere la recuperación de políticas económicas activas orientadas al desarrollo del territorio y el diseño de proyectos para emprendedores que exploten estrategias de cooperación entre actividades económicas de diferentes sectores de actividad para promover el crecimiento económico y social desde una perspectiva sostenible. Es de evidenciar la relevancia que estas políticas activas requieren sobre el territorio y la necesidad que las áreas de desarrollo local recuperen el protagonismo y capacidades necesarias para atender a la complejidad actual con la intención de promocionar el mismo a partir de sus potencialidades ociosas o infrautilizadas.

\section{Métodos}

Para esta investigación se utilizará el método deductivo que nos permitirá interpretar y comprender las particularidades del tema planteado.

Se utilizarán la investigación documental y descriptiva considerando que tenemos como objeto de estudio la producción de soya en el Ecuador y sus oportunidades en el mercado mundial. La Investigación descriptiva "Se reseñan la características o rasgos de la situación o fenómeno objeto del estudio" (Bernal, Cesar A, 2010) y la Investigación Documental "La investigación documental consiste en un análisis de la información escrita sobre un determinado tema, con el propósito de establecer relaciones, diferencias, etapas, posturas o estado actual del conocimiento respecto al tema objeto del estudio." (Bernal, Cesar A, 2010). A través de la obtención de la información para la investigación por medio de la observación, internet y análisis de documental del tema planteado y a través de los datos obtenidos de la página de páginas web.

\section{Resultados}

La necesidad del desarrollo local. Un breve repaso terminológico Intentar intuir cómo se va a comportar el futuro de la economía es complejo y, en muchas ocasiones, incierto. Pero sí parece más evidente, la distancia que se va imponiendo entre lo particular y lo global. Esta distancia es la que se debe compensar de alguna manera para que lo particular pueda aprehender su capacidad de desarrollo. Dentro de este ámbito particular se engloba el desarrollo local y sus políticas activas sustentadas sobre los recursos endógenos, las capacidades territoriales y la necesidad de entender el territorio como una unidad económica completa que compite con otras para poder sobrevivir ( (Cantarero, 2011)). 
El termino industria proviene del latín industrius que revela actividad productiva, laboriosidad, asiduidad, encomio, en general dícese de actividades de provecho encaminadas a cumplir un objetivo.

En el desarrollo de la historia de las economías este término ha sido utilizado para denominar a las actividades creadoras de valor, en la creación de mercancías que serían destinadas para la obtención de una renta. La palabra productividad, así se ha venido refinando su connotación hasta la actualidad en que se utiliza para referirse a aspectos diversos, dentro de la economía de un país en la creación de su riqueza, como en particular al referirse propiamente dentro de una industria en el específico sentido al analizar su capacidad de generación de riqueza es decir la producción.

\section{Al respecto en la legislación ecuatoriana se considera en el (Código Orgánico de la Producción, Comercio e Inversiones)}

Título Preliminar - Del Objetivo y Ámbito de Aplicación Art. 4

c. Fomentar la producción nacional, comercio y consumo sustentable de bienes y servicios, con responsabilidad social y ambiental, así como su comercialización y uso de tecnologías ambientalmente limpias y de energías alternativas; 23

h. Regular la inversión productiva en Sectores estratégicos de la economía, de acuerdo AL Plan Nacional de Desarrollo;

La corriente de cambios tendientes a la recesión económica que experimentan las economías, ha dado paso a que el ingenio, creatividad en inventiva de los emprendedores se oriente a crear nuevas propuestas de empresas que atiendan a necesidades también propias de esta nueva sociedad en la que se da prioridad a la conservación del medio ambiente y se prima la responsabilidad con el medio ambiente y el respeto a su desarrollo en armonía con el ser humano.

Al tratar de nuevas ideas de negocios tocamos el emprendimiento que según (Schumpeter, 1934) El emprendedor es "El fundador de una nueva empresa, un innovador que rompe la forma tradicional de hacer las cosas, con rutinas establecidas. Debe ser una persona con dotes de liderazgo, y con un talento especial para identificar el mejor modo de actuar.”

También es tendencia en el cambiante ritmo que emprendimiento las tendencias de mercado que la innovación es motor del desarrollo económico, las empresas maximizan sus rendimientos económicos al apostar por la combinación innovación y mostrar respeto por la sustentabilidad del medio ambiente.

\section{Antecedentes}

El arte clásico de los griegos en la línea del tiempo de la humanidad, enseña a plasmar la belleza en esculturas madera, marfil, mármol, oro y plata, porque tenían la necesidad de capturar en sus obras las expresiones y movimientos de lo que consideraban bello, así Platón escribió: si «Hay algo por lo que vale la pena vivir, es por contemplar la belleza». (Platón, 427 AC-347 AC.) 
Mientras tanto en otro punto del tiempo, en la prehistoria tiene la necesidad de transportar artículos por su condición de nómadas, y así surgen los bolsos o bolsas desarrollado por los hombres y mujeres de esa época. Esta misma necesidad se trasporta al siglo XVII apareciendo prendas pequeñas, donde hombres y mujeres guardaban sus cartas y documentos personales.

Pero es la unión de esa necesidad y belleza que da origen al Balandrán o retículo (retículus - ridicule ${ }^{1}$ ) para guardar pañuelos y pequeños objetos de la época y desde entonces la evolución de los bolsos y prendas está dado por cada época, circunstancia y necesidad.

En el 2013 el Museo Nacional de Baviera, en el sur de Alemania organizó un recorrido por la cultura de prendas, desde el siglo XVI al XIX con 300 piezas históricas (Universal, 2013). Las prendas son más que un accesorio, son una forma de expresión que identifica cada etapa de una vida dado por los cambios sociales de una época, como en 1789 cuando la mujer toma conciencia por primera vez y de manera colectiva de su situación social marchando hacia Versalles para reclamar "Libertad, igualdad y fraternidad" (Gouge, 1791).

\section{Problemas y oportunidades}

¿Qué tan realizable es crear y posesionar una empresa y emprendimiento de diseño, corte y confección de prendas para el género femenino entre 7 y 65 años de edad, para que sea aceptada y sostenible en el sector artesanal? $(\mathbf{C A N})$ :

En primer lugar, se debe definir el tipo de empresa según las ventas determinados por la

\begin{tabular}{|c|c|c|}
\hline $\begin{array}{l}\text { Clasificación de las } \\
\text { empresas }\end{array}$ & $\begin{array}{l}\text { Volúmenes de } \\
\text { ventas anuales }\end{array}$ & $\begin{array}{l}\text { Personal } \\
\text { ocupado }\end{array}$ \\
\hline Micro empresa & Menor a 100.000 & $1 \mathrm{~A} 9$ \\
\hline Pequeña empresa & $\begin{array}{l}\text { De } 100.001 \mathrm{a} \\
1^{\prime} 000.000\end{array}$ & 10 A 49 \\
\hline "A" Mediana empresa & $\begin{array}{l}\text { De 1’000.001 a } \\
2^{\prime} 000.000\end{array}$ & 50 A 99 \\
\hline $\begin{array}{l}\text { Mediana empresa } \\
\text { "B" }\end{array}$ & $\begin{array}{l}\text { De 2’000.001 a } \\
5^{\prime} 000.000\end{array}$ & 100 A 199 \\
\hline Grande empresa & $\begin{array}{l}\text { De 5'000.001 en } \\
\text { adelante }\end{array}$ & $\begin{array}{l}200 \text { en } \\
\text { adelante }\end{array}$ \\
\hline
\end{tabular}

INEC, Ecuador en cifras, 2014 
Según el (INEC, 2014): Quito posee entre micros, pequeñas, medianas y grandes 201.629 empresas, seguida por el Guayas con 159.937, Manabí 69.611, Azuay 49.026, Tungurahua 43.036, el oro 41.643, Los Ríos 29.291, Loja 28.629, Chimborazo 28.471, Imbabura 27.288, Cotopaxi 25.798, santo domingo de los Tsáchilas 22.545, Esmeraldas 18.173, Cañar 15.579 ,Bolívar 11.763 Santa Elena 11.298, Carchi 10.649, Sucumbíos 9.659, Orellana 7.910, Zamora Chinchipe 7.821, Napo 7.480, Morona Santiago 6.963, Pastaza 6.572 y Galápagos 2.872.

La industria manufacturera $^{2}$ al 2014 posee el $8.1 \%$ del mercado, es decir, 68.095 empresas. Su concentración según la clasificación de las empresas está dada por: micro empresa $8 \%$, pequeña empresa $8.8 \%$, mediana empresa " $\mathrm{A}$ " $8.8 \%$, mediana empresa "B" $9.3 \%$ y grande empresa 15.7\%. El total de ventas \$34.342.035.873, es decir, 20.3\% del mercado. Sin embargo, analizar el volumen de ventas de las microempresas en el sector manufacturero artesanal, que es la categorización donde pertenece la empresa, es excluido en las fuentes estadísticas, por la ausencia de información en un 95\% de los casos, debido a que su actividad es artesanal, está conformado principalmente de personas naturales no obligadas a llevar contabilidad y pertenecientes al RISE según él (SRI, 2014).

A pesar de que existen varías curtidurías en Ecuador, ubicadas principalmente en la región sierra o interandina, y de que hay muchos artesanos realizando bienes finales a base de cuero, este se exporta más como materia prima al mercado europeo que como bien final, al menos en el caso de las prendas. No obstante el sector del cuero ha experimentado índices de crecimiento sostenido gracias a las políticas gubernamentales con respecto a las importaciones y el estímulo a los productores nacionales para producir bienes finales, en este trabajo se va a presentar otras alternativas de materia prima basándose en las exigencias y preferencias de mercado globales, manteniendo similares estándares de calidad con fibras sintéticas o semi naturales como: microfibras, media piel, eco piel (vegana y animal, curtida y tintada con minerales o vegetales) o poli piel (brillante y labrada, metalizada e impresa) que sean benefactores con el medio ambiente y acapare con todos los mercados posibles, por ejemplo: grupos ecologistas.

La exclusividad e individualización como estrategia de innovación para los modelos que se diseñarán, será una tarea compartida y divertida donde las personas tendrán la oportunidad de elegir entre colores, texturas y diseños, para confeccionar su prenda ideal. Esto será a través de un asesoramiento calificado y eficiente. Sin embargo, el mercado ecuatoriano valora mucho en los grandes emprendimientos su tradición, tiempo y origen, lo cual significa un reto para posesionarse en el mercado.

\section{Objetivo general}

Orientar y diseñar nuevos modelos artesanales de corte, confección y diseños personalizados y exclusivos de prendas, bolsos y demás, para distribuir a nivel nacional e internacional.

\section{Objetivo específico}

\footnotetext{
${ }^{2}$ Proceso de fabricación de un producto que se realiza con las manos o con ayuda de máquinas, a partir de una materia prima
} 
Analizar los sectores, real, externo y tributario del país de origen y el de exportación

Categorizar por edad, ingresos, preferencias y uso la producción

Asesorar la imagen de cada mujer, a través de los modelos, colores, texturas y tamaños adecuado para cada una

Distinguir el servicio de la individualización de las prendas

Organizar dos eventos al año, como lanzamiento de nuevas colecciones

\section{Estudio de Mercado}

Para establecer el precio de las prendas basándose en la demanda y oferta del país de origen (Ecuador) se ha tomado en cuenta el salario básico unificado (SBU) que en el país es de $\$ 366$ desde el 2016. Además del dominicano donde será el destino a exportar, en el que existen 3 categorías: salario mínimo mayor RD\$12,873.00 (281,11 USD); segundo salario RD\$8,850.00 (193,26 USD); y, tercer salario RD\$7,843.00 (171,27 USD).

Sin embargo, antes de eso es importante entender la segmentación de mercado femenino dispuesto a adquirir prendas y bolsos en ambos países, que estará dado de la siguiente forma:

El mercado femenino de forma fija está dispuesto a pagar entre \$20 y \$50 dólares inicialmente por prenda acogiendo esta tendencia eco amigable al menos dos veces al año, en las fechas más comerciales, esto se daría entre la festividad mundial black Friday y navidad.

Sin embargo, todo el año las mujeres compran prendas, su frecuencia se determinará a través de una encuesta en algunos sectores de la ciudad de Guayaquil.

\section{Oferta}

Bajo la condición ceteris paribus asumiendo el mismo nivel de demanda diaria, la oferta de la empresa sería de 30 unidades cada día, 150 semanales y 600 mensuales de prendas entre las de mano, pequeñas, medianas y grandes, trabajando 8 horas por día y 20 días al mes.

\section{Demanda}

Un elemento fundamental de una buena planeación de la comercialización consiste en pronosticar con la mayor exactitud la demanda de un producto. El pronóstico de la demanda es estimar las ventas de un producto durante determinado periodo futuro. 


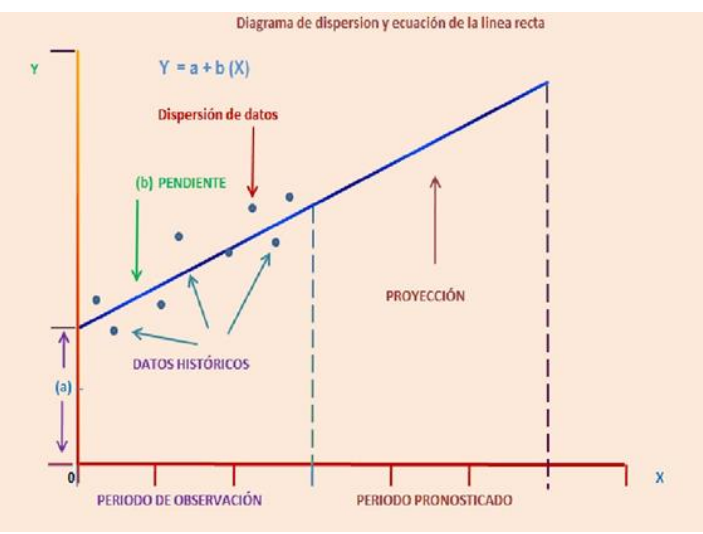

Pronósticos de demanda-Regresión Lineal

El año 1 equivale al 2016, en el cual se vendieron 240 prendas anuales (20 prendas mensuales por 12 meses del año), con una sola persona durante 8 horas diarias por 20 días al mes. Entre el 2017 y 2024 se prevé un crecimiento del 4\% anual de la demanda.

Demanda pronosticada con método de regresión lineal

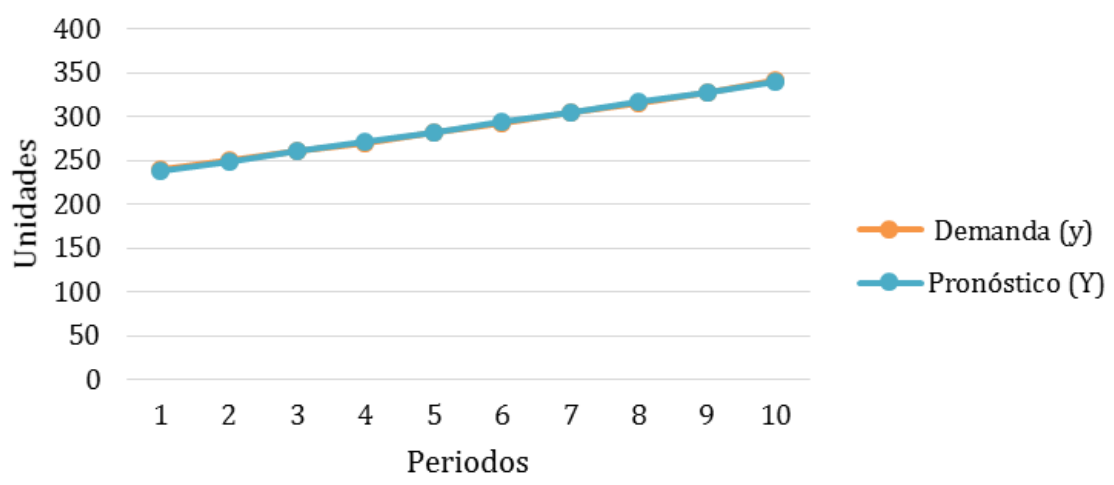

\section{Localización}

En ecuador estará ubicada en la ciudad de Guayaquil inicialmente, al tener facilidad de acceso con El Puerto Marítimo de la ciudad, que está localizado en la costa occidental de América del Sur, en un brazo del mar, el Estero Salado, a diez Kilómetros al Sur del centro comercial de la ciudad del mismo nombre siendo éste el más importante punto geográfico de la costa oeste de Sudamericana.

\section{Puerto de Guayaquil, 2016}

Sin embargo, a partir del 16 de febrero del 2016, La empresa Dubaití DP Word oficializó su inversión de \$1.200 millones para el proyecto del Puerto de Aguas Profundas de Posorja ya que su ubicación estratégica está cerca de las rutas navieras internacionales y estaciones con tiempo de pilotaje mínimo, lo cual le da competitividad. Así que este cambio geográfico afectará positivamente la economía en Posorja por lo que se deberá analizar en las etapas de 
prefactibilidad y factibilidad los factores que van a afectar a este proyecto: aspectos legales, insumos, sondeo de mercado (consumidores y formas de distribución), impacto ambiental, entre otros, para definir finalmente si conviene permanecer en Guayaquil o trasladar la empresa a Posorja.

Para elegir el lugar definitivo para la empresa se debe decidir si se quiere estar cerca de la materia prima, la cual es importada y el nivel de producción que tendríamos en ambos lugares, para determinar en cual se tendría más beneficios y menos costos.

\section{Exportación a república dominicana}

Principales características de las condiciones de acceso físico desde ecuador al mercado de destino en término de los diferentes medios de transporte vía marítima, acceso al mar

a) Puertos, nombres, ubicaciones, capacidad de almacenaje, áreas de influencia, calado y conexión con carreteras.

Los puertos comerciales de cierto alcance se limitan al de Puerto Plata en el norte, y los de la capital, San Pedro de Macorís y Barahona en la costa sur.

El principal en tráfico mercantil es Haina. El más moderno y segundo en movimiento de mercancías es el multimodal privado de Caucedo, de propiedad privada. Ambos, al igual que el de Boca Chica, especializados en contenedores, se encuentran muy próximos a la capital. Hay también un pequeño puerto en la propia ciudad de Santo Domingo para carga y pasajeros. Otros más pequeños con tráfico de cruceros turísticos son Samaná y La Romana.

\section{El puerto de Río Haina}

Es en la actualidad el principal y más concurrido puerto en la República Dominicana. Alrededor del $70 \%$ de toda la carga se mueve a través de este, situado a sólo 6 millas de distancia de Santo Domingo de la ciudad capital. Hay instalaciones portuarias en las dos orillas del río Haina, al oeste del poblado del mismo nombre en la latitud $18^{\circ} 25 \mathrm{~N}$, longitud $70^{\circ} 00 \mathrm{~W}$. Con un calado de 10 metros ( 28 a 35 pies), con una profundidad en el canal de entrada de 10.67 metros (35 pies), un circulo de maniobra de 300 metros (984 Pies) y una longitud de muelle de 3,044 metros (10,000 Pies).

No realiza operaciones de buques de pasajeros, actualmente está concesionado a la empresa Haina Internacional Terminal HIT. En el mismo se manejan carga general, granos, madera, palanquilla, carga contenedorizada, y combustible.

\section{DP World Caucedo}

Es una terminal marítima y zona franca de clase mundial localizada en Punta Caucedo, a 25 kilómetros de la ciudad de Santo Domingo, capital comercial y política del país. Forma parte del porta-folio de DP World, líder internacional en operaciones, desarrollo de nuevas terminales portuarias, logística y servicios relacionados. Este puerto privado inició sus operaciones en diciembre del 2003 y cuenta con tecnología de punta para el manejo de operaciones portuarias. 
Además, dispone de los sistemas de seguridad más avanzados de la industria y una combinación única en el mundo de certificaciones de seguridad adquiridas provee instalaciones para embarcaciones modernas Post-Panamá y Súper Post Panamá.

\section{Puerto de Boca Chica}

Este puerto se encuentra localizado en la bahía de Andrés Boca Chica en la latitud $18^{\circ} 27$ $\mathrm{N}$ y longitud $69^{\circ} 35 \mathrm{~W}$ con un calado de 30 pies, con una longitud de muelle de 615 metros (2,017.2 Pies), un circulo de maniobra de 200 metros (656 Pies), un ancho en el canal de entrada de 120 metros (393.6 Pies) con una profundidad de 8.54 metros (28 Pies), y un atracadero en forma de L.

Este puerto fue construido en los años 50 en la época del auge azucarero. Al principio solo existía un atracadero para el embarque de azúcar que se transportaba en vagones de ferrocarril desde el ingenio hasta los barcos surtos en el mismo.

Actualmente sirve para operaciones de buques comerciales de exportación e importación, cargas en contenedores subproductos de papel y carga general.

En este puerto se encuentra una barcaza generadora de electricidad.

b) Líneas navieras que operan en el lugar, tarifas, frecuencias y rutas

La ruta Guayaquil- Caucedo se ofrece directo en 16 días costo aproximado por contenedor de 40 pies HC USD 1,700.00, el mismo que es sujeto a negociación entre las partes contratantes.

\section{Vía terrestre. Red de carreteras, principales terminales, líneas}

\section{Red de carreteras}

Una red de carreteras de $19.705 \mathrm{Km}$, de las cuales $9.872 \mathrm{Km}$ están pavimentadas y 9.833 sin pavimentar, y $260 \mathrm{Km}$. de carretera de peaje, destacando el tramo Santo Domingo-aeropuerto y la carretera de Santo Domingo a Samaná. Las cuatro arterias principales absorben la mayoría del tráfico y se hallan en un estado razonable de mantenimiento.

Hay, además, alrededor de $14.000 \mathrm{Km}$. de caminos vecinales, de los que están asfaltados menos del $10 \%$ de su trazado.

\section{Vía aérea}

El aeropuerto internacional que más pasajeros recibe, debido a los vuelos chárter, es Punta Cana. El de Las Américas, que sirve a la capital, va en segundo lugar. Siguen en importancia Puerto Plata, Santiago, La Romana, Samaná y Barahona.

a) Principales aeropuertos, tarifas, frecuencias, líneas y rutas

\section{Aeropuerto de Punta Cana}

El aeropuerto internacional de Punta Cana se ubica $30 \mathrm{~km}$ (18 millas) al sur de los complejos y resorts de Punta Cana. Es uno de los más importantes del Caribe en cuanto a tráfico 
internacional y el más transitado de República Dominicana. Opera con varios destinos internacionales como ser: los principales puntos de Estados Unidos, Canadá, México, toda la región del Caribe y Centroamérica, Sudamérica y Europa.

Debido al acelerado crecimiento de tráfico, se encuentra en continua expansión. Cuenta con la particularidad de un techo de palma, totalmente abierto y realmente turístico. Alrededor de 4.46 millones de pasajeros lo transitaron en 2011.

\section{Aeropuerto de Las Américas}

El aeropuerto internacional de Santo Domingo, Las Américas, se ubica en Punta Caucedo, a $30 \mathrm{~km}$ (18 millas) al sureste del centro de Santo Domingo.

Es uno de los principales aeropuertos de República Dominicana y uno de los más importantes de América Central y el Caribe. Opera con varios destinos internacionales como ser: los principales puntos de Estados Unidos, Canadá, México, toda la región del Caribe y Centroamérica, Sudamérica y Europa. Alrededor de 3.07 millones de pasajeros lo transitaron en 2011.

\section{Aeropuerto de Puerto Plata}

El aeropuerto internacional de Puerto Plata se ubica $18 \mathrm{~km}$ (11 millas) al este de Puerto Plata es uno de los más importantes de República Dominicana.

Opera con varios destinos internacionales como son: los principales puntos de Estados Unidos, Canadá, gran parte del Caribe y Centroamérica.

Alrededor de 440 mil pasajeros lo transitaron en 2010.

\section{Documentos para aduana}

Es necesario conocer de los convenios internacionales suscritos entre el país y el resto de zonas comerciales. Bajo los (INCOTERMS, 2010) los más utilizados son FOB para exportaciones y CIF para importaciones.

Sin embargo, como alternativa para envíos de carácter urgente, de exportaciones en volúmenes pequeños y muestras sin valor comercial, se encuentran los servicios de Courier, los cuales garantizan mejores tiempos de entrega.

\begin{tabular}{|l|}
\hline \multicolumn{1}{|c|}{ Documentos de Importación } \\
\hline Conocimiento de embarque \\
\hline Orden de liberación de carga \\
\hline Certificado de origen \\
\hline Factura Comercial \\
\hline Declaración Unica Aduanera \\
\hline Lista de empaque \\
\hline Recibos de manipulación en terminal \\
\hline
\end{tabular}

\begin{tabular}{|l|}
\hline \multicolumn{1}{|c|}{ Documentos de Exportación } \\
\hline Conocimiento de embarque \\
\hline Certificado de origen \\
\hline Factura Comercial \\
\hline Declaración de exportación \\
\hline Lista de empaque \\
\hline Inspección de pre embarque \\
\hline Doing Business
\end{tabular}

\section{Documentos exigibles}


Tiempos y costos de los trámites de exportación e importación según doing bussines en República Dominicana:

\begin{tabular}{|l|r|r|}
\hline \multicolumn{1}{|c|}{ Procedimiento de Exportación } & Duracion (dias) & USS Costo \\
\hline Preparación de documentos & 3 & 215 \\
\hline Control aduanero & 2 & 200 \\
\hline Manejo terminal portuario & 1 & 325 \\
\hline Transporte interno & 2 & 300 \\
\hline Total & 8 & 1,040 \\
\hline Doing Business
\end{tabular}

\begin{tabular}{|l|r|r|}
\hline \multicolumn{1}{|c|}{ Procedimiento de Importación } & Duración (dias) & \multicolumn{1}{c|}{ USS Costo } \\
\hline Preparación de documentos & 5 & 240 \\
\hline Control aduanero & 2 & 200 \\
\hline Manejo terminal portuario & 2 & 410 \\
\hline Transporte interno & 1 & 300 \\
\hline Total & 10 & 1,150 \\
\hline
\end{tabular}

(Business, s.f.)

El objetivo de es vender el emprendimiento de la empresa como franquicia, sin embargo, en el corto plazo asumiendo que se tiene el primer comprador en República Dominicana al por menor o mayor y habiendo cumplido con los documentos mencionados, es necesario destacar la información que debe contener la factura, esta se debe acordar los términos de compra venta en un contrato Internacional. En este documento se debe indicar claramente las características del producto, la cantidad demandada, el medio de transporte, los plazos de entrega, el lugar de entrega del producto, la forma de pago y si existe un adelanto de pago.

El contrato debe reflejar el acuerdo logrado en las negociaciones y se debe tener cuidado con la forma de cotización entregada y el término de comercio internacional (INCOTERMS, 2010) utilizado debido a que tienen diferentes niveles de obligaciones, costos y riesgos para ambas partes.

En la factura comercial se indica el término o condiciones de pago, descripción, cantidad, vendedor y consignatario de la mercancía, así como él (INCOTERMS, 2010).

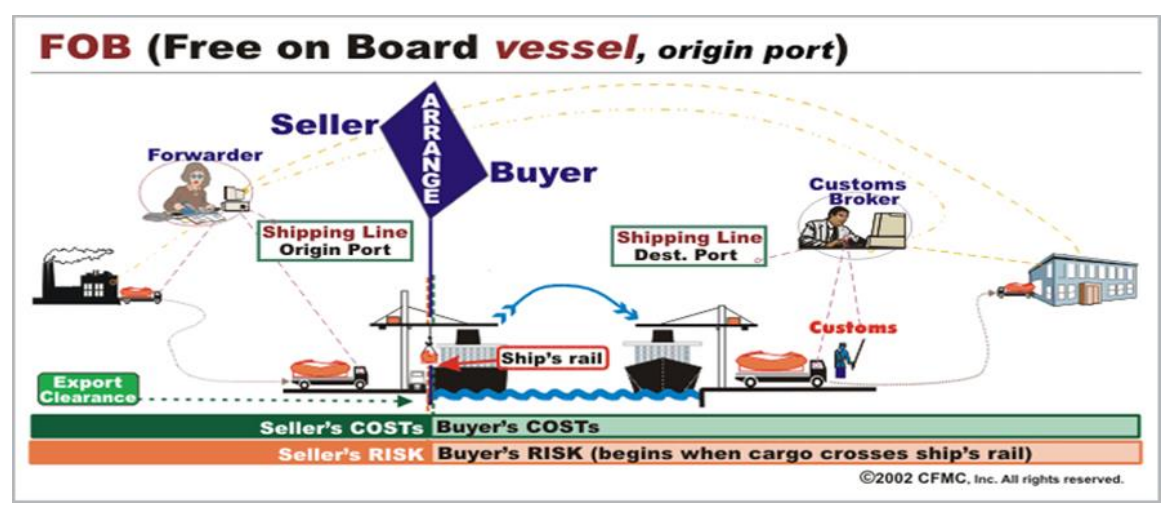




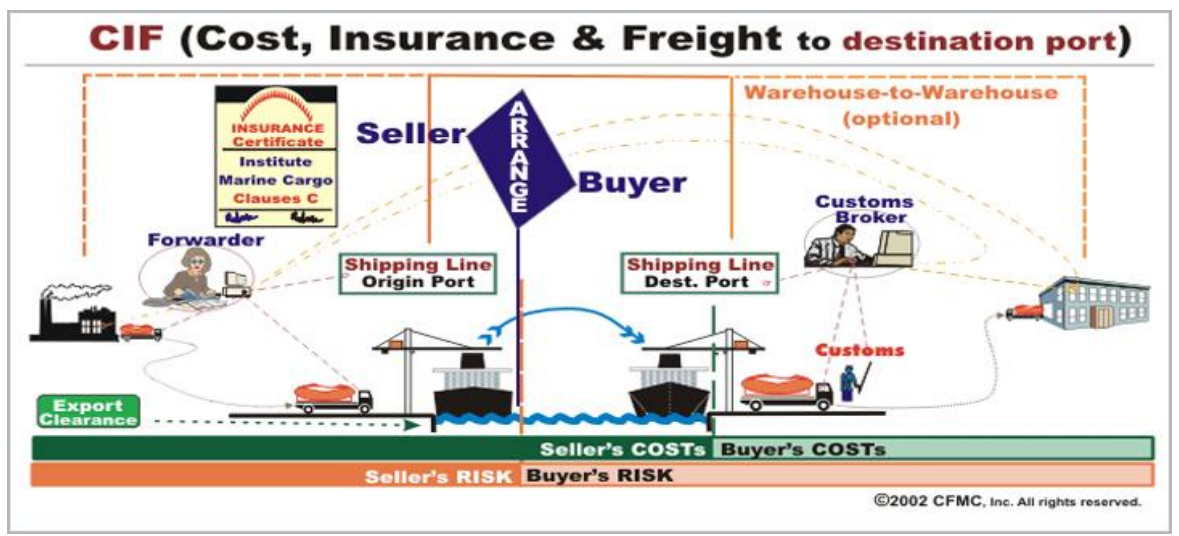

(CFMC, 2010)

\section{Conclusiones}

Las oportunidades de emprendimiento son propicias para implementar una empresa artesanal de diseño, corte y confección de prendas para el género femenino producido con fibras sintéticas o semi naturales resistentes a las manchas, de alta durabilidad y densidad, facilidad de limpieza y superficie libre de pelusas.

Se contará con algunas líneas para el género femenino, segmentándolos en primer lugar por: edad, ingresos, preferencias, uso, entre otros.

La industria textil abarca todas las actividades de fabricación obtención de materias primas para la creación de fibras, hilar, tejer, entintar y darles acabados distinguidos propios de la confección de prendas según el segmento de mercado que estén dirigidas.

La diseño, confección y personalización del producto se ejecutará usando material importado de fibras sintéticas, la mano de obra será nacional y estará capacitada para usar máquinas de coser industriales de costura recta. Se busca abarcar todo el mercado femenino principalmente entre las siete y sesenta y cinco años de edad a través del emprendimiento. Esta se caracterizará por exclusividad, individualización y creatividad como pilares esenciales para la aceptación y valoración del producto.

La incumbencia laboral, social y ambiental son los siguientes estribos que fortalecerán el emprendimiento, dadas por las exigencias gubernamentales, sociales y como base de los principios morales y éticos para asegurar el éxito correcto de la empresa.

En Ecuador aún queda mucho por innovar en el mercado textil, al poseer acceso al comercio internacional a través de su privilegiada posición geográfica, bien puede servir como generador de mercancías y/o una vez posicionada la marca de un nuevo emprendimiento, realizar su maquia mediante franquiciado, y servir como eje de negociaciones y centro de distribución estratégico para llegar a los mercados de los países de América y de Europa. 


\section{Bibliografía}

Bernal, Cesar A. (2010). Metodología de la Investigación. En B. Cesar, Metodología de la Investigación. Bogotá: Orlando Fernández.

Bernal, Cesar A. (2010). Metodología de la Investigación. En C. A. BERNAL, Metodología de la investigación (pág. 111). Bogotá: Orlando Fernandez.

Business, D. (s.f.). www.doingbusiness.com.

CAN, C. A. (s.f.).

Cantarero. (2011).

Censos, I. N. (2016). Encuesta Nacional de empleo, desempleo y subempleo en el Ecuador.

CFMC. (2010). www.cfcm.com.

Código Orgánico de la Producción, Comercio e Inversiones. (s.f.). Código Orgánico de la Producción, Comercio e Inversiones.

Gouge, O. d. (1791). Declaración de los derechos de la mujer y de la ciudadana.

INCOTERMS. (2010).

INEC. (2014).

Platón. (427 AC-347 AC.). Filósofo. Belleza y Estética. Gracia.

Schumpeter. (1934).

SRI, S. d. (2014).

Universal, E. (martes de abril de 2013). El Universal. Obtenido de El Universal: http://archivo.eluniversal.com.mx/notas/916992.html 